\title{
Evaluación de la definición clínica de dengue sugerida por la Organización Mundial de la Salud
}

\begin{abstract}
Ruth Aralí Martínez, Fredi Alexander Díaz, Luis Angel Villar
Centro de Investigaciones Epidemiológicas, Facultad de Salud, Universidad Industrial de Santander, Bucaramanga, Colombia.

Introducción. Clínicamente es difícil diferenciar el dengue de otras entidades con sintomatología similar.

Objetivo. Evaluar la utilidad diagnóstica de la definición de caso presunto de dengue sugerida por la Organización Mundial de la Salud, en un área endémica (Bucaramanga, Colombia). Materiales y métodos. En una cohorte con síndrome febril agudo sin causa aparente (mayores de 12 años), se determinó la sensibilidad, la especificidad y los valores pronósticos de dicha definición que incluye dos o más de estas manifestaciones: cefalea, dolor retroocular, mialgias, artralgias, exantema, manifestaciones hemorrágicas y leucopenia.

Resultados. Se incluyeron 101 pacientes de dengue, confirmados serológica o virológicamente, y 89 con síndrome febril agudo de otra etiología. La definición de caso exhibió una sensibilidad de 99\%; especificidad de 1\%; valor pronóstico positivo de 53\%, y valor pronóstico negativo de $50 \%$.

Conclusión. Esta definición clínica es sensible, pero poco específica; por tanto, puede ser útil para tamizaje, pero no ayuda a diferenciar el dengue de otras enfermedades febriles.
\end{abstract}

Palabras clave: dengue, OMS, sensibilidad, especificidad, valores pronósticos.

\section{Evaluation of the World Health Organization clinical definition of dengue}

Introduction.The distinction between dengue and other acute febrile diseases is difficult when based solely on symptoms.

Objective. An attempt was made to evaluate usefulness of dengue diagnoses in an endemic area (Bucaramanga, Colombia), based on the definitions recommended by the World Health Organization.

Materials and methods. In a cohort $>12$ years of age with indications of acute febrile illness, the sensitivity, the specificity and the predictive values were determined based on the WHO dengue definition. Two or more of the following symptoms were included: headache, retroorbital pain, myalgia, arthralgia, rash, hemorrhagic manifestations, or leucopenia.

Results. One hundred-one cases of dengue and 89 patients with acute febrile illness were enrolled in the study. The dengue cases were confirmed by serological or by virus presence. The WHO case definitions showed sensitivity, 99\%; specificity,1\%; positive predictive value, $53 \%$, and negative predictive value, $50 \%$.

Conclusion. This clinical definition demonstrated high sensitivity, but a low specificity. Therefore, the clinical definition was useful for screening, but it did not differentiate between dengue and other febrile diseases.

Keywords: dengue, WHO, sensitivity, specificity, predictive values.

Correspondencia:

Ruth Aralí Martínez, Carrera 32 No. 29-31, tercer piso. Bucaramanga, Colombia.

Telefax: (577) 6345781

rutharam@yahoo.com

Recibido: 11/02/05; aceptado: 10/05/05
El dengue es la arbovirosis humana que causa mayor morbilidad y mortalidad a nivel mundial (1). Se estima que más de 100 millones de personas se infectan con este virus cada año, por lo que se ha convertido en un importante problema de salud pública (1-3). 
En Colombia, el dengue es una enfermedad endemo-epidémica en zonas por debajo de los 1.800 metros sobre el nivel del mar y gran parte de nuestra población está en riesgo de presentarla $(4,5)$. En el departamento de Santander, al noreste del país, las tasas de incidencia se han incrementado en los últimos años y han oscilado entre 113,4 y 268,7 casos por 100.000 habitantes $(4,5)$.

Para establecer presuntivamente el diagnóstico de dengue, según la definición de la Organización Mundial de la Salud (OMS), el paciente debe presentar fiebre asociada a dos o más de las siguientes manifestaciones: cefalea, dolor retroocular, mialgias, artralgias, exantema, manifestaciones hemorrágicas y leucopenia (6).

La utilidad de esta definición para establecer el diagnóstico de dengue en pacientes febriles puede tener limitaciones, principalmente, las derivadas de la dificultad clínica para distinguir esta arbovirosis de entidades que se inician como un síndrome febril agudo inespecífico y se acompañan de varios de los síntomas que la OMS considera en su definición. Hacen parte del diagnóstico diferencial enfermedades como influenza, rubéola, gastroenteritis, fiebre tifoidea y leptospirosis, entre otras $(1,3,7,8)$.

El dengue presenta, además, variabilidad de sus síntomas y signos, por lo que se ha cuestionado el uso de una definición dependiente del cumplimiento de un reducido número de criterios clínicos y se ha hecho énfasis en la confirmación serológica o virológica de la enfermedad $(9,10)$. Sin embargo, los resultados de estas pruebas no están disponibles en los primeros días de la enfermedad, cuando se requiere de un diagnóstico temprano para establecer pautas de manejo.

Así, aun cuando es deseable contar con herramientas clínicas para el diagnóstico del dengue, éstas deben ser altamente sensibles y específicas. El presente estudio evalúa la utilidad diagnóstica de la definición presuntiva de dengue establecida por la OMS, a partir de una cohorte de pacientes con síndrome febril agudo de etiología no establecida, en un área endémica para esta arbovirosis.

\section{Metodología}

Diseño: estudio de evaluación de prueba diagnóstica en una cohorte concurrente.

Población: individuos mayores de doce años que consultaron a centros de salud del área metropolitana de Bucaramanga (Colombia), durante el periodo de abril del 2003 a enero del 2004, con síndrome febril agudo de causa no aparente y a quienes el médico les hizo inicialmente una impresión diagnóstica de dengue, "virosis" o infección viral no especificada, o fiebre no especificada (códigos de la Clasificación estadística internacional de enfermedades $y$ problemas relacionados con la salud, décima revisión (CIE-10): A90X, B349 y R509, respectivamente).

Criterios de inclusión: pacientes con menos de 96 horas de enfermedad (consideradas a partir del inicio de la fiebre) y aceptación de la participación en el estudio mediante su firma (o la de su acudiente) del consentimiento informado, previamente revisado y aprobado por el Comité de Ética de la Universidad Industrial de Santander.

Criterios de exclusión: se excluyeron los pacientes que al momento del ingreso hubieran presentado alguna de las siguientes condiciones:

1. Evidencia clínica de otro proceso infeccioso que explique de forma parcial o total la enfermedad actual.

2. Diabetes, sida, cirrosis, enfermedades reumatológicas o tumorales, insuficiencia cardiaca o renal, o historia de ingestión de corticoides.

3. Residencia en zona rural o de difícil acceso para el seguimiento.

Evaluación y seguimiento: a cada individuo se le realizó una valoración inicial que incluyó una anamnesis y un examen físico realizado por un médico, con una prueba de torniquete hecha siguiendo los lineamientos de la OMS (6). Todos los signos y síntomas evaluados se registraron en un formato individual estandarizado.

Después de la valoración clínica, entre las 48 y 96 primeras horas de enfermedad, se tomó una 
muestra de sangre para realizar un cuadro hemático automatizado y obtener suero (agudo) para aislamiento viral y medición de anticuerpos IgM contra dengue. Después del séptimo día de enfermedad, se tomó una segunda muestra de suero (convaleciente) para realizar una nueva medición de anticuerpos.

Para la determinación de los niveles de IgM se utilizó un sistema de ensayo de captura (MACELISA) adaptado en el Laboratorio de Virología del Centro de Investigaciones en Enfermedades Tropicales de la Universidad Industrial de Santander (CINTROP, UIS). La modificación incluida fue descrita por Innis et al. (11). Las muestras de suero se diluyeron hasta una relación de 1:40 y se evaluaron con un antígeno tetravalente de dengue (CDC, Puerto Rico). La prueba se consideró positiva cuando el valor de absorbancia fue, al menos, el doble de la media de los controles negativos. El aislamiento viral se hizo en cultivo de células de mosquito Aedes albopictus (clon C6/36) empleando las muestras de fase aguda que permanecieron almacenadas a $-70^{\circ} \mathrm{C}$ hasta el momento del cultivo.

Definición de caso: se consideraron casos de dengue aquéllos en los que se evidenció una seroconversión (IgM aguda negativa e IgM convaleciente positiva), una cuadriplicación de los títulos de IgM o un aislamiento viral positivo. Cuando la prueba de IgM específica contra dengue en la convalecencia fue negativa, se consideró que el síndrome febril agudo tenía otra etiología (síndrome febril aguda no dengue).

Almacenamiento y análisis de datos: la información fue almacenada en una base de datos electrónica (Microsoft Excel 97, Microsoft Corp). Se describió la frecuencia de los signos y síntomas de la definición de caso presunto de dengue de la OMS en cada grupo de pacientes (dengue y síndrome febril agudo no dengue). Se estimó la utilidad diagnóstica de la definición clínica de dengue y de sus componentes (incluso, la prueba de torniquete como manifestación específica), determinando su sensibilidad (S), especificidad (E) y valores pronósticos positivo y negativo (VPP y VPN). Además, se evaluó la aplicación de definiciones que reunieran más de dos manifestaciones clínicas.

\section{Resultados}

De 190 pacientes incluidos, 101 (53,2\%) fueron casos de dengue y 89 de síndrome febril agudo no dengue. La proporción de hombres fue de $51,5 \%$ (52) y $49,4 \%$ (44), respectivamente. La media de edad fue similar entre los grupos (media \pm DE: $28,67 \pm 15,345$ vs. $29,06 \pm 14,328$ ).

La cefalea, las mialgias y las artralgias fueron manifestaciones muy frecuentes en los pacientes con dengue; sin embargo, la especificidad de estos síntomas fue inferior al 20\%. Por otra parte, el

Cuadro 1. Sensibilidad, especificidad y valores pronósticos de las manifestaciones que conforman la definición de caso de dengue de la OMS en una cohorte de adultos (mayores de 12 años) con síndrome febril aguda.

\begin{tabular}{lcccccc}
\hline Manifestación & $\begin{array}{c}\text { dengue } \\
(\mathbf{n = 1 0 1 )}\end{array}$ & $\begin{array}{c}\text { no dengue } \\
(\mathbf{n = 8 9 )}\end{array}$ & $\mathbf{S ~ ( \% )}$ & E (\%) & VPP (\%) & VPN (\%) \\
\hline Cefalea & 97 & 84 & 96,04 & 5,62 & 53,59 & 55,56 \\
Mialgias & 95 & 73 & 94,06 & 11,24 & 54,60 & 62,5 \\
Artralgias & 86 & 72 & 85,15 & 19,10 & 54,43 & 53,13 \\
Dolor retroocular & 72 & 57 & 71,29 & 35,96 & 55,81 & 52,46 \\
Exantema & 48 & 17 & 47,52 & 80,90 & 73,85 & 57,6 \\
Manifestación hemorrágica * & 53 & 33 & 52,47 & 62,92 & 61,63 & 53,38 \\
Prueba de torniquete positiva $\dagger$ & $44 / 100$ & 27 & 44,00 & 69,70 & 61,97 & 52,54 \\
Hemorragia espontánea & 15 & 8 & 14,85 & 91,01 & 65,22 & 48,50 \\
Leucopenia $\ddagger$ & 89 & 47 & 88,12 & 47,19 & 65,44 & 77,78 \\
\hline
\end{tabular}

* Incluye hemorragias espontáneas 0 , al menos, una prueba de torniquete positiva.

† Se consideró positiva cuando se observaron más de 20 petequias en una pulgada cuadrada, luego de mantener un torniquete en la media de la presión arterial durante 5 minutos (OMS). Un paciente con dengue no aceptó la realización de la prueba.

‡ Recuento de leucocitos menor de $5.000 / \mathrm{mm}^{3}$ 
exantema y las manifestaciones hemorrágicas aunque mostraron una sensibilidad menor, presentaron los mayores valores de especificidad (cuadro 1).

La definición de caso presunto de dengue de la OMS tuvo una alta sensibilidad, pero su especificidad fue muy baja. La aplicación de definiciones más exigentes, reuniendo un mayor número de manifestaciones clínicas, aumentó progresivamente su especificidad y VPP (cuadro 2).

La sensibilidad, la especificidad y los VPP y VPN de una definición exclusivamente clínica, con dos o más síntomas sin tener en cuenta el dato de la leucopenia, fueron similares a las obtenidas cuando se adicionó este parámetro de laboratorio (S, 98\%; E, 2\%; VPP, 53\%; VPN, 50\%).

\section{Discusión}

Nuestro estudio confirma la alta prevalencia del dengue en una población con síndrome febril aguda en un área endémica. Sin embargo, el 46,8\% de los pacientes sufren otras causas de enfermedad febril con manifestaciones clínicas similares a las del dengue. Un ejemplo de ello podría ser el virus de la influenza, un frecuente patógeno, que junto con el dengue son las principales causas de síndrome febril agudo de origen inespecífico en diferentes regiones del mundo $(12,13)$.

En otros estudios también se han encontrado limitaciones para identificar los casos de dengue a partir de una definición clínica $(10,14)$. En El Salvador, la evaluación de una descripción clínica de dengue reveló un VPP de sólo el 14\% (14). En
Brasil, la definición clínica de la OMS mostró una $S$ de $64 \%$ y se asoció a $57 \%$ de falsos positivos (10). Estos hallazgos y los del presente estudio sugieren que el reporte de casos basado únicamente en la descripción clínica puede sobreestimar notablemente la incidencia de dengue, aun en poblaciones de alta endemicidad.

Los resultados del presente estudio demuestran una excelente sensibilidad de las manifestaciones que componen la definición clínica de la OMS, pero también indican que éstas, de forma individual, difícilmente ayudarían a diferenciar el dengue de otras entidades. Por otra parte, las manifestaciones hemorrágicas (espontáneas y la prueba de torniquete) son hallazgos con mejor especificidad diagnóstica, como se reportó en otro estudio (15).

La reunión de varios síntomas mejoraría la $\mathrm{E}$ y el VPP de la descripción clínica, al punto que un paciente con 6 o más manifestaciones tendría una probabilidad de $79 \%$ de presentar dengue (VPP), con una especificidad de $85 \%$. Una definición con, al menos, cinco manifestaciones también ofrece una especificidad y VPP aceptables (cuadro 2).

La alta sensibilidad de la definición de la OMS (con 2 o más manifestaciones) la convierte en una herramienta útil para el tamizaje de pacientes con síndrome febril agudo que consultan en los servicios de urgencias. Sin embargo, dada su baja especificidad, la aplicación de esta definición en la vigilancia epidemiológica del dengue, sin la ayuda de estudios serológicos, llevaría a sobreestimar la incidencia de la enfermedad. En ausencia de pruebas de laboratorio que confirmen

Cuadro 2. Sensibilidad, especificidad y valores pronósticos positivo y negativo de la descripción clínica de dengue en una cohorte de pacientes con síndrome febril aguda.

\begin{tabular}{lcccrrr}
\hline $\begin{array}{c}\text { Número de manifestaciones } \\
\text { aplicadas * }\end{array}$ & $\begin{array}{c}\text { dengue } \\
(\mathbf{n = 1 0 1 )}\end{array}$ & $\begin{array}{c}\text { no dengue } \\
(\mathbf{n = 8 9 )}\end{array}$ & $\mathbf{S ~ ( \% )}$ & E (\%) & VPP (\%) & VPN (\%) \\
\hline 2 o más † & 100 & 88 & 99,01 & 1,12 & 53,19 & 50,00 \\
3 o más & 98 & 81 & 97,03 & 8,99 & 54,75 & 72,73 \\
4 o más & 93 & 74 & 92,08 & 16,85 & 55,69 & 65,22 \\
5 o más & 51 & 25 & 50,50 & 71,91 & 67,11 & 56,14 \\
6 o 7 & 49 & 13 & 48,52 & 85,39 & 79,03 & 59,38 \\
\hline
\end{tabular}

* Se tuvieron en cuenta las siguientes manifestaciones: cefalea, dolor retroocular, mialgias, artralgias, exantema, manifestaciones hemorrágicas y leucopenia.

† Corresponde a la definición recomendada por la OMS. 
el diagnóstico, sería recomendable considerar como caso de dengue, aquéllos que reúnan un mayor número de manifestaciones (por ejemplo, al menos, 5).

\section{Conflicto de interés}

Los autores declaramos que no existe un posible conflicto de intereses en este manuscrito.

\section{Agradecimientos}

Por su colaboración en la realización de las pruebas diagnósticas, agradecemos a Raquel Elvira Ocazionez, Fabián Cortés y Sergio Gómez del Centro de Investigaciones en Enfermedades Tropicales (CINTROP), y a Karol Torres del Laboratorio Clínico de la Universidad Industrial de Santander.

\section{Financiación}

Este estudio está anidado en la cohorte de pacientes del proyecto 1102-04-12919, cofinanciado por Colciencia y la Universidad Industrial de Santander.

\section{Referencias}

1. Gubler DJ. Dengue and dengue hemorrhagic fever. Clin Microbiol Rev 1998;11:480-96.

2. Tesh RB. Viral hemorrhagic fevers of South America. Biomedica 2002;22:287-95.

3. Levett PN, Branch SL, Edwards CN. Detection of dengue infection in patients investigated for leptospirosis in Barbados. Am J Trop Med Hyg 2000;62:112-4.

4. Ministerio de Salud. Comportamiento por regiones del dengue en el 2001. Boletín Epidemiológico Semanal, SIVIGILA. Semana epidemiológica No. 02. Enero 06 a 12 de 2002. www.col.ops-oms.org/sivigila/2002/ BOLE02_02.htm

5. Ministerio de Salud. Enfermedades de notificación obligatoria. Informe primer semestre de 2003. Boletín Epidemiológico Semanal, SIVIGILA. Semana epidemiológica No. 26. Junio 22-28 de 2003. www.col.ops-oms.org/sivigila/2003/BOLE26_03.pdf
6. Organización Panamericana de la Salud Definiciones de casos. Dengue. Boletín Epidemiológico 2000;21:14-5.

7. Watt G, Jongsakul K, Chouriyagune C, Paris R. Differentiating dengue virus infection from scrub typhus in Thai adults with fever. Am J Trop Med Hyg 2003;68: 536-8.

8. Seijo A, Cernigoi B, Deodato B. Dengue importado de Paraguay a Buenos Aires. Reporte clínico y epidemiológico de 38 casos. Medicina (Buenos Aires) 2001;61:137-41.

9. Pan American Health Organization. Dengue and dengue hemorrhagic fever in the Americas: guidelines for prevention and control. Washington, D.C.: PAHO; 1994. p.3-22.

10. Dietz VJ, Gubler DJ, Rigau-Perez JG, Pinheiro F, Schatzmayr HG, Bailey R et al. Epidemic dengue 1 in Brazil, 1986: evaluation of a clinically based dengue surveillance system. Am J Epidemiol 1990;131:693-701.

11. Innis BL, Nisalak A, Nimmannitya $S$, Kusalerdchariya S, Chongswasdi V, Suntayakorn $\mathrm{S}$ et al. An enzyme-linked immunosorbent assay to characterize dengue infections where dengue and Japanese encephalitis co-circulate. Am J Trop Med Hyg 1989;40:418-27.

12. Instituto Nacional de Salud de Colombia, Ministerio de Salud. Epidemia de influenza A/H3N2/Beijing/32/92 en Ambalema, Tolima, 1994. Inf Quinc Epidemiol Nac 1996;1:6-8

13. Silarug N, Foy HM, Kupradinon S, Rojanasuphot S, Nisalak A, Pongsuwant Y. Epidemic of fever of unknown origin in rural Thailand, caused by influenza $A$ (H1N1) and dengue fever. Southeast Asian J Trop Med Public Health 1990;21:61-7.

14. Hayes JM, Garcia-Rivera E, Flores-Reyna R, SuarezRangel G, Rodriguez-Mata T, Coto-Portillo R et al. Risk factors for infection during a severe dengue outbreak in El Salvador in 2000. Am J Trop Med Hyg 2003;69:629-33.

15. Kalayanarooj S, Vaughn DW, Nimmannitya S, Green $\mathrm{S}$, Suntayakorn S, Kunentrasai $\mathrm{N}$ et al. Early clinical and laboratory indicators of acute dengue illness. J Infect Dis 1997;176:313-21. 